\title{
Signal Processing by Generalized Receiver in DS-CDMA Wireless Communication Systems with Optimal Combining and Partial Cancellation
}

\author{
Vyacheslav Tuzlukov \\ School of Electronics Engineering, College of IT Engineering, Kyungpook National University, Room 407A, Building IT3, \\ 1370 Sankyuk-dong, Buk-gu, Daegu 702-701, Republic of Korea
}

Correspondence should be addressed to Vyacheslav Tuzlukov, tuzlukov@ee.knu.ac.kr

Received 2 June 2010; Revised 25 November 2010; Accepted 5 February 2011

Academic Editor: Kostas Berberidis

Copyright ( 2011 Vyacheslav Tuzlukov. This is an open access article distributed under the Creative Commons Attribution License, which permits unrestricted use, distribution, and reproduction in any medium, provided the original work is properly cited.

Symbol error rate (SER) of quadrature subbranch hybrid selection/maximal-ratio combining (HS/MRC) scheme for 1-D modulations in Rayleigh fading under employment of the generalized receiver (GR), which is constructed based on the generalized approach to signal processing (GASP) in noise, is investigated. $N$ diversity input branches are split into $2 N$ inphase and quadrature subbranches. $M$-ary pulse amplitude modulation, including coherent binary phase-shift keying (BPSK), with quadrature subbranch HS/MRC is investigated. GR SER performance for quadrature HS/MRC and HS/MRC schemes is investigated and compared with the conventional HS/MRC receiver. Comparison shows that the GR with quadrature subbranch $\mathrm{HS} / \mathrm{MRC}$ and HS/MRC schemes outperforms the traditional HS/MRC receiver. Procedure of partial cancellation factor (PCF) selection for the first stage of hard-decision partial parallel interference cancellation (PPIC) using GR employed by direct-sequence code-division multiple access (DS-CDMA) systems under multipath fading channel in the case of periodic code scenario is proposed. Optimal PCF range is derived based on Price's theorem. Simulation confirms that the bit error rate (BER) performance is very close to potentially achieved one and surpasses the BER performance of real PCF for DS-CDMA systems discussed in the literature.

\section{Introduction}

In this paper, we investigate the generalized receiver (GR), which is constructed based on the generalized approach to signal processing (GASP) in noise [1-5], under quadrature subbranch hybrid selection/maximal-ratio combining (HS/MRC) for 1-D modulations in multipath fading channel and compare its symbol error rate (SER) performance with that of the traditional HS/MRC scheme discussed in $[6,7]$. It is well known that the HS/MRC receiver selects the $L$ strongest signals from $N$ available diversity branches and coherently combines them. In a traditional HS/MRC scheme, the strongest $L$ signals are selected according to signalenvelope amplitude [6-12]. However, some receiver implementations recover directly the in-phase and quadrature components of the received branch signals. Furthermore, the optimal maximum likelihood estimation (MLE) of the phase of a diversity branch signal is implemented by first estimating the in-phase and quadrature branch signal components and obtaining the signal phase as a derived quantity $[13,14]$. Other channel-estimation procedures also operate by first estimating the in-phase and quadrature branch signal components [15-18]. Thus, rather than $N$ available signals, there are $2 \mathrm{~N}$ available quadrature branch signal components for combining. In general, the largest $2 L$ of these $2 N$ quadrature branch signal components will not be the same as the $2 L$ quadrature branch signal components of the $L$ branch signals having the largest signal envelopes.

In this paper, we investigate how much improvement in performance can be achieved employing the GR with modified HS/MRC, namely, with quadrature subbranch HS/MRC and HS/MRC schemes, instead of the conventional 
HS/MRC combining scheme for 1-D signal modulations in multipath fading channel. At GR discussed in [19], the $N$ diversity branches are split into $2 N$ in-phase and quadrature subbranches. Then, the GR with HS/MRC scheme is applied to these $2 \mathrm{~N}$ subbranches. Obtained results show the better performance is achieved by this quadrature subbranch HS/MRC scheme in comparison with the traditional HS/MRC scheme for the same value of average signal-tonoise ratio (SNR) per diversity branch.

Another problem discussed is the problem of partial cancellation factor (PCF) in a DS-CDMA system with multipath fading channel. It is well known that the multiple access interference (MAI) can be efficiently estimated by the partial parallel interference cancellation (PPIC) procedure and then partially be cancelled out of the received signal on a stage-by-stage basis for a direct-sequence code-division multiple access (DS-CDMA) system [20]. To ensure a good performance, PCF for each PPIC stage needs to be chosen appropriately, where the PCF should be increased as the reliability of the MAI estimates improves. There are some papers on the selection of the PCF for a receiver based on the PPIC. In [21-23], formulas for the optimal PCF were derived through straightforward analysis based on soft decisions. In contrast, it is very difficult to obtain the optimal PCF for a receiver based on PPIC with hard decisions owing to their nonlinear character. One common approach to solve the nonlinear problem is to select an arbitrary PCF for the first stage and then increase the value for each successive stage, since the MAI estimates may become more reliable in later stages $[20,24,25]$. This approach is simple, but it might not provide satisfactory performance.

In this paper, we use Price's theorem [26, 27] to derive a range of the optimal PCF for the first stage in PPIC of DS-CDMA system with multipath fading channel employing GR based on GASP [1-5], where the lower and upper boundary values of the PCF can be explicitly calculated from the processing gain and the number of users of DS-CDMA system in the case of periodic code scenario. Computer simulation shows that, using the average of these two boundary values as the PCF for the first stage, we are able to reach the bit error rate (BER) performance that is very close to the potentially achieved one [28] and surpasses the BER performance of the real PCF for DS-CDMA systems discussed, for example in [20].

With this result, a reasonable PCF can quickly be determined without using any time-consuming Monte Carlo simulations. It is worth mentioning that the two-stage GR considered in [29] based on the PPIC using the proposed $\mathrm{PCF}$ at the first stage achieves the BER performance comparable to that of the three-stage GR based on the PPIC using an arbitrary PCF at the first stage. In other words, at the same BER performance, the proposed approach for selecting the PCF can reduce the GR complexity based on the PPIC. The PCF selection approach is derived for multipath fading channel cases discussed in $[19,30]$.

The paper is organized as follows. In Section 2, we describe the multipath fading channel model and provide system models for selection/maximal ratio combining and synchronous DS-CDMA, and recall the main functioning principles of GR. We carry out the performance analysis in Section 3 where we obtain a symbol error rate expression in the closed form and define a marginal moment generating function of SNR per symbol of a single quadrature branch. In Section 4, we determine the lower and upper PCF bounds based on the processing gain $N$ and the number of users $K$ under multipath fading channel model in DS-CDMA system employing GR. Finally, simulation results are given in Section 5, and some conclusions are made in Section 6.

\section{System Model}

2.1. Multipath Fading Channel Model. Let the transfer function for user $k^{\prime}$ s channel be

$$
W_{k}(Z)=\sum_{i=1}^{M} \alpha_{k, i} Z^{-\tau_{k, i}} .
$$

As we can see from (1), the number of paths is $M$ and the channel gain and delay for $i$ th channel path are $\alpha_{k, i}$ and $\tau_{k, i}$, respectively. We use two vectors to represent these parameters:

$$
\begin{aligned}
\boldsymbol{\tau}_{k} & =\left[\tau_{k, 1}, \tau_{k, 2}, \ldots, \tau_{k, L}\right]^{T}, \\
\boldsymbol{\alpha}_{k} & =\left[\alpha_{k, 1}, \alpha_{k, 2}, \ldots, \alpha_{k, L}\right]^{T} .
\end{aligned}
$$

Let

$$
\tau_{k, 1} \leq \tau_{k, 2} \leq \cdots \leq \tau_{k, L}
$$

and the channel power is normalized

$$
\sum_{i=1}^{L} \alpha_{k, i}^{2}=1 .
$$

Without loss of generality, we may assume that $\tau_{k, 1}=0$ for each user and $L$ is the maximum possible number of paths. When a user's path number, say $M_{1}$, is less than $M$, we can let all the elements in $\tau_{k, i}$ and $\alpha_{k, i}$ be zero if the following condition is satisfied

$$
M_{1}+1 \leq i \leq M
$$

We may also assume that the maximum delay is much smaller than the processing gain $N$ [23]. Before our formulation, we first define a $(2 N-1) \times L$ composite signature matrix $\mathbf{A}_{k}$ in the following form

$$
\mathbf{A}_{k}=\left[\widetilde{\mathbf{a}}_{k, 1}, \widetilde{\mathbf{a}}_{k, 2}, \ldots, \widetilde{\mathbf{a}}_{k, L}\right],
$$

where $\widetilde{\mathbf{a}}_{k, i}$ is a vector containing $i$ th delayed spreading code for user $k$. It is defined as

$$
\tilde{\mathbf{a}}_{k, i}=[\overbrace{0, \ldots, 0}^{\tau_{k, i}}, \mathbf{a}_{k}^{T}, \overbrace{0, \ldots, 0}^{N-\tau_{k, i}-1}]^{T} .
$$

Since a multipath fading channel is involved, the current received bit signal will be interfered by previous bit signal. As mentioned above, the maximum path delay is much smaller than the processing gain. The interference will not be severe and for simplicity, we may ignore this effect. Let us denote the channel gain for multipath fading as

$$
\mathbf{h}_{k}=\boldsymbol{\alpha}_{k} \mathbf{A}_{k}
$$




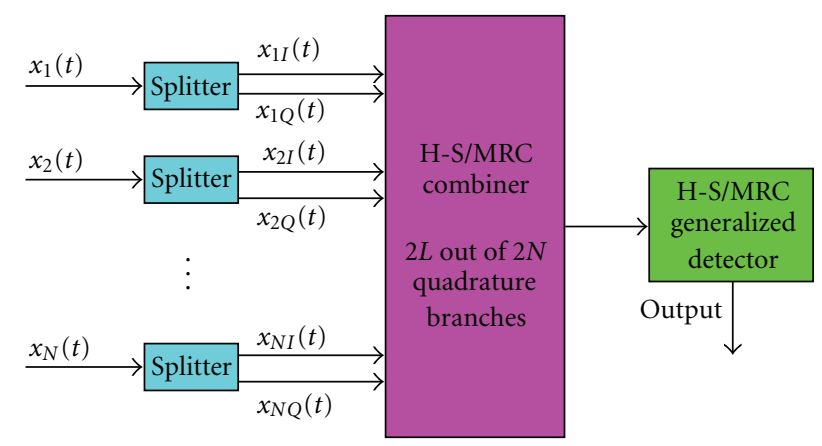

FIgURE 1: Block diagram receiver based on GR with quadrature subbranch HS/MRC and HS/MRC schemes.

2.2. Selection/Maximal-Ratio Combining. We assume that there are $N$ diversity branches experiencing slow and flat Rayleigh fading, and all of the fading processes are independent and identically distributed (i.i.d.). During analysis, we consider only the hypothesis $H_{1}$ "a yes" signal in the input stochastic process. Then the equivalent received baseband signal for the $k$ th diversity branch takes the following form:

$$
x_{k}(t)=h_{k}(t) s\left(t-\tau_{k}\right)+n_{k}(t), \quad k=1, \ldots, N,
$$

where $s\left(t-\tau_{k}\right)$ is a $1-\mathrm{D}$ baseband transmitted signal that, without loss of generality, is assumed to be real, $h_{k}(t)$ is the complex channel gain for the $k$ th branch subjected to Rayleigh fading, $\tau_{k}$ is the propagation delay along the $k$ th path of the received signal, and $n_{k}(t)$ is a zero-mean complex AWGN with two-sided power spectral density $N_{0} / 2$ with the dimension $\mathrm{W} / \mathrm{Hz}$. At GR front end, for each diversity branch, the received signal is split into its in-phase and quadrature signal components. Then, the conventional HS/MRC scheme is applied over all of these quadrature branches, as shown in Figure 1.

We can present $h_{k}(t)$ given by (1)-(8) as i.i.d. complex Gaussian random variables assuming that each of the $L$ branches experiences slow, flat, Rayleigh fading

$$
h_{k}(t)=\alpha_{k}(t) \exp \left\{-j \varphi_{k}(t)\right\}=\alpha_{k} \exp \left\{-j \varphi_{k}\right\}
$$

where $\alpha_{k}$ is a Rayleigh random variable and $\varphi_{k}$ is a random variable uniformly distributed within the limits of the interval $[0,2 \pi)$. Owing to the fact that the fade amplitudes are Rayleigh distributed, we can present $h_{k}(t)$ as

$$
h_{k}(t)=h_{k I}(t)+j h_{k Q}(t)
$$

and $n_{k}(t)$ as

$$
n_{k}(t)=n_{k I}(t)+j n_{k Q}(t) .
$$

The in-phase signal component $x_{k I}(t)$ and quadrature signal component $x_{k Q}(t)$ of the received signal $x_{k}(t)$ are given by

$$
\begin{gathered}
x_{k I}(t)=h_{k I}(t) a\left(t-\tau_{k}\right)+n_{k I}(t), \\
x_{k Q}(t)=h_{k Q}(t) a\left(t-\tau_{k}\right)+n_{k Q}(t) .
\end{gathered}
$$

Since $h_{k}(t)(k=1, \ldots, K)$ are subjected to i.i.d. Rayleigh fading, we can assume that the in-phase $h_{k I}(t)$ and quadrature $h_{k Q}(t)$ channel gain components are independent zero-mean Gaussian random variables with the same variance [18]

$$
\sigma_{h}^{2}=\frac{1}{2} \mathrm{E}\left[\left|h_{k}^{2}(t)\right|\right]
$$

where $\mathrm{E}[\cdot]$ is the mathematical expectation. Further, the inphase $n_{k I}(t)$ and quadrature $n_{k Q}(t)$ noise components are also independent zero-mean Gaussian random processes, each with two-sided power spectral density $N_{0} / 2$ with the dimension $\mathrm{W} / \mathrm{Hz}$ [13]. Due to the independence of the in-phase $h_{k I}(t)$ and quadrature $h_{k Q}(t)$ channel gain components and the in-phase $n_{k I}(t)$ and quadrature $n_{k Q}(t)$ noise components, the $2 N$ quadrature branch received signal components conditioned on the transmitted signal are i.i.d.

We can reorganize the in-phase and quadrature components of the channel gains $h_{k}$ and Gaussian noise $n_{k}(t)$ when $k=1, \ldots, N$ as $g_{k}$ and $v_{k}$, given, respectively, by

$$
\begin{aligned}
& g_{k}(t)= \begin{cases}h_{k I}(t), & k=1, \ldots, N, \\
h_{(k-N) Q}(t), & k=N+1, \ldots, 2 N ;\end{cases} \\
& v_{k}(t)= \begin{cases}n_{k I}(t), & k=1, \ldots, N, \\
n_{(k-N) Q}(t), & k=N+1, \ldots, 2 N .\end{cases}
\end{aligned}
$$

The GR output with quadrature subbranch HS/MRC and HS/MRC schemes according to GASP [1-5] is given by

$$
\begin{aligned}
Z_{\mathrm{QBHS} / \mathrm{MRC}}^{g}(t)= & s^{2}(t) \sum_{k=1}^{2 N} c_{k}^{2} g_{k}^{2}(t) \\
& +\sum_{k=1}^{2 N} c_{k}^{2} g_{k}^{2}(t)\left[v_{k_{\mathrm{AF}}}^{2}(t)-v_{k_{\mathrm{PF}}}^{2}(t)\right],
\end{aligned}
$$

where $v_{k_{\mathrm{AF}}}^{2}(t)-v_{k_{\mathrm{PF}}}^{2}(t)$ is the background noise forming at the GR output for the $k$ th branch; $c_{k} \in\{0,1\}$ and $2 L$ of the $c_{k}$ equal 1.

2.3. Generalized Receiver (GR). For better understanding (17), we recall the main functioning principles of GR. The simple model of GR in form of block diagram is represented in Figure 2. In this model, we use the following notations: MSG is the model signal generator (local oscillator), the AF is the additional filter (the linear system), and the PF is the preliminary filter (the linear system). A detailed discussion of the AF and PF can be found in [2, pages 233-243 and 264284] and [5]. Consider briefly the main statements regarding the $\mathrm{AF}$ and $\mathrm{PF}$.

There are two linear systems at the GR front end that can be presented, for example, as bandpass filters, namely, the $\mathrm{PF}$ with the impulse response $h_{\mathrm{PF}}(\tau)$ and the AF with the impulse response $h_{\mathrm{AF}}(\tau)$. For simplicity of analysis, we think that these filters have the same amplitude-frequency responses and bandwidths. Moreover, a resonant frequency of the AF is detuned relative to a resonant frequency of $\mathrm{PF}$ on such a value that signal cannot pass through the AF (on a value that is higher the signal bandwidth). Thus, the signal 


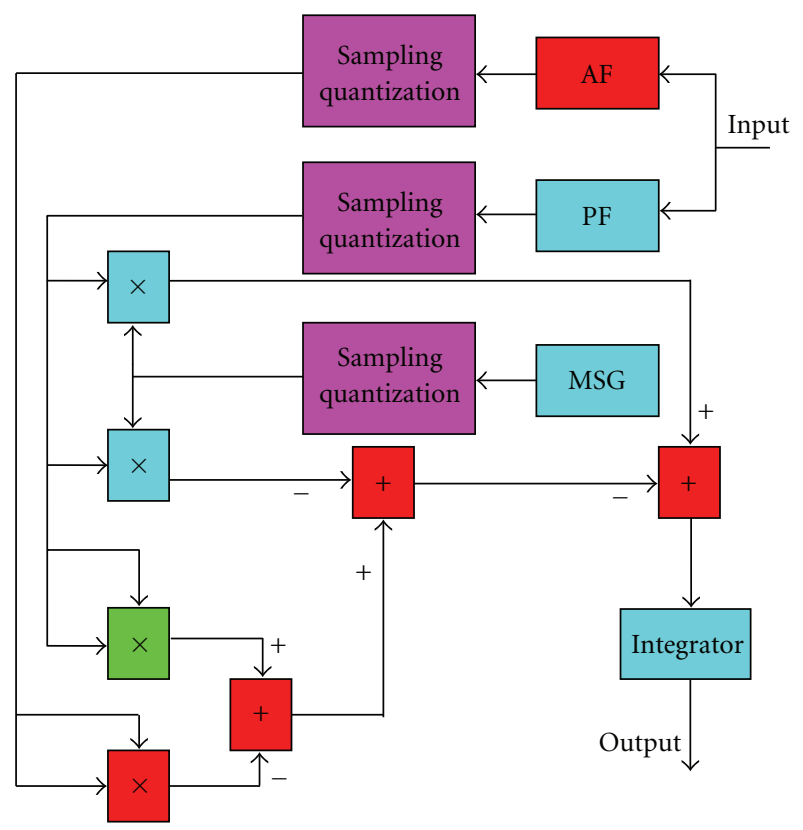

FIGURE 2: Principal block diagram model of the GR.

and noise can be appeared at the PF output and the only noise is existed at the AF output. It is well known, if a value of detuning between the $\mathrm{AF}$ and $\mathrm{PF}$ resonant frequencies is more than $4 \div 5 \Delta f_{a}$, where $\Delta f_{a}$ is the signal bandwidth, the processes forming at the AF and PF outputs can be considered as independent and uncorrelated processes (in practice, the coefficient of correlation is not more than 0.05 ).

In the case of signal absence in the input process, the statistical parameters at the AF and PF outputs will be the same, because the same noise is coming in at the AF and $\mathrm{PF}$ inputs, and we may think that the AF and PF do not change the statistical parameters of input process, since they are the linear GR front end systems. For this reason, the AF can be considered as a generator of reference sample with $a$ priori information a "no" signal is obtained in the additional reference noise forming at the AF output.

There is a need to make some comments regarding the noise forming at the PF and AF outputs. If the Gaussian noise $n(t)$ comes in at the AF and PF inputs (the GR linear system front end), the noise forming at the AF and PF outputs is Gaussian, too, because the AF and PF are the linear systems and, in a general case, takes the following form:

$$
\begin{aligned}
& v_{k_{\mathrm{PF}}}(t)=\int_{-\infty}^{\infty} h_{\mathrm{PF}}(\tau) v_{k}\left(t-\tau_{k}\right) d \tau, \\
& v_{k_{\mathrm{AF}}}(t)=\int_{-\infty}^{\infty} h_{\mathrm{AF}}(\tau) v_{k}\left(t-\tau_{k}\right) d \tau .
\end{aligned}
$$

If, for example, AWGN with zero mean and two-sided power spectral density $N_{0} / 2$ is coming in at the AF and PF inputs (the GR linear system front end), then the noise forming at the AF and PF outputs is Gaussian with zero mean and variance given by [4, pages 264-269]

$$
\sigma_{n}^{2}=\frac{N_{0} \omega_{0}^{2}}{8 \Delta_{F}}
$$

where, in the case the AF (or PF) is the RLC oscillatory circuit, the AF (or PF) bandwidth $\Delta_{F}$ and resonance frequency $\omega_{0}$ are defined in the following manner

$$
\Delta_{F}=\pi \beta, \quad \omega_{0}=\frac{1}{\sqrt{L C}}, \quad \text { where } \beta=\frac{R}{2 L} .
$$

The main functioning condition of GR is the equality over the whole range of parameters between the model signal $s_{k}^{*}(t)$ at the GR MSG output for user $k$ and expected signal $s_{k}(t)$ forming at the GR input liner system (the PF) output, that is,

$$
s_{k}(t)=s_{k}^{*}(t)
$$

How we can satisfy this condition in practice is discussed in detail in [2, pages 669-695] and [5]. More detailed discussion about a choice of PF and AF and their amplitudefrequency responses is given in $[2,5]$ (see also http://www .sciencedirect.com/science/journal/10512004, click "Volume 8, 1998", "Volume 8, Issue 3", and "A new approach to signal detection theory").

2.4. Synchronous DS-CDMA System. Consider a synchronous DS-CDMA system employing the GR with $K$ users, the processing gain $N$, the number of frame $L$, the chip duration $T_{c}$, the bit duration $T_{b}=N T_{c} / R$ with information bit encoding rate $R$. The signature waveform of the user $k$ is given by

$$
a_{k}(t)=\sum_{i=1}^{N} a_{k i} p_{T_{c}}\left(t-i T_{c}\right),
$$

where $\left\{a_{k 1}, a_{k 2}, \ldots, a_{k N}\right\}$ is a random spreading code with each element taking value on $\pm 1 / \sqrt{N}$ equiprobably, $p_{T_{c}}(t)$ is the unit-amplitude rectangular pulse with duration $T_{c}$. The baseband signal transmitted by the user $k$ is given by

$$
s_{k}(t)=A_{k}(t) \sum_{i=1}^{L} b_{k, i} a_{k}\left(t-i T_{b}\right),
$$

where $A_{k}(t)$ is the transmitted signal amplitude of the user $k$.

The following form can present the received baseband signal:

$$
\begin{aligned}
x(t) & =\sum_{k=1}^{K} h_{k}(t) s_{k}(t)+n(t) \\
& =\sum_{k=1}^{K} \sum_{i=1}^{L} S_{k}(t) b_{k, i} a_{k}\left(t-i T_{b}\right)+n(t), \quad t \in\left[0, T_{b}\right],
\end{aligned}
$$

where, taking into account (1)-(8) and (10) and as it was shown in [31],

$$
S_{k}(t)=h_{k}(t) A_{k}(t)=\alpha_{k}^{2} A_{k}(t)
$$


is the received signal amplitude envelope for the user $k, n(t)$ is the complex Gaussian noise with zero mean with

$$
E\left[n_{k}(t)\left(n_{j}(t)\right)^{*}\right]= \begin{cases}2 \alpha_{k}^{2} \sigma_{n}^{2}, & \text { if } j=k \\ 2 \alpha_{k}^{2} \sigma_{n}^{2} \rho_{k j}, & \text { if } j \neq k,\end{cases}
$$

$\rho_{k j}$ is the coefficient of correlation.

Using GR based on the multistage PPIC for DS-CDMA systems and assuming the user $k$ is the desired user, we can express the corresponding GR output according to GASP (see Figure 2) and the main functioning condition of GR given by (21) as the first stage of the PPIC GR:

$$
\begin{aligned}
Z_{k}(t)= & \int_{0}^{T_{b}}\left[2 x_{k}(t) s_{k}^{*}\left(t-\tau_{k}\right)-x_{k}(t) x_{k}\left(t-\tau_{k}\right)\right] d t \\
& +\int_{0}^{T_{b}} \alpha_{k}^{2} v_{k_{\mathrm{AF}}}(t) v_{k_{\mathrm{AF}}}\left(t-\tau_{k}\right) d t
\end{aligned}
$$

where $s_{k}^{*}(t)$ is the model of the signal transmitted by the user $k$ (see $(21)$ ); $\tau_{k}$ is the delay factor that can be neglected for simplicity of analysis. For this case, we have

$$
\begin{aligned}
Z_{k} & =S_{k}(t) b_{k}+\sum_{j=1, j \neq k}^{K} S_{j}(t) b_{j} \rho_{k j}+\zeta_{k} \\
& =S_{k}(t) b_{k}+I_{k}(t)+\zeta_{k}(t) \\
& =h_{k}(t) A_{k}(t) b_{k}+I_{k}(t)+\zeta_{k}(t),
\end{aligned}
$$

where the first term in (28) is the desired signal,

$$
\rho_{k j}=\int_{0}^{T_{b}} s_{k}(t) s_{j}(t) d t
$$

is the coefficient of correlation between signature waveforms of the $k$ th and $j$ th users; the third term in(28)

$$
\zeta_{k}=\int_{0}^{T_{b}} \alpha_{k}^{2}\left[v_{k_{\mathrm{AF}}}^{2}(t)-v_{k_{\mathrm{PF}}}^{2}(t)\right] d t
$$

is the total noise component at the GR output; the second term in(28)

$$
\begin{aligned}
I_{k} & =\sum_{j=1, j \neq k}^{K} S_{j} b_{j} \rho_{k j} \\
& =\sum_{j=1, j \neq k}^{K} h_{j} A_{j} b_{j} \rho_{k j} \\
& =\sum_{j=1, j \neq k}^{K} \alpha_{j}^{2} A_{j} b_{j} \rho_{k j}
\end{aligned}
$$

is the MAI. The conventional GR makes a decision based on $Z_{k}$. Thus, MAI is treated as another noise source. When the number of users is large, MAI will seriously degrade the system performance. GR with partial interference cancellation, being a multiuser detection scheme [8], is proposed to alleviate this problem.
Denoting the soft and hard decisions of the GR output for the user $k$ by

$$
\tilde{b}_{k}^{(0)}=Z_{k}, \quad \hat{b}_{k}^{(0)}=\operatorname{sgn}\left(Z_{k}\right),
$$

respectively, the output of the GR with the first PPIC stage with a partial cancellation factor equal to $p_{1}$ can be written as [20]

$$
\begin{aligned}
\tilde{b}_{k}^{(1)} & =p_{1}\left(Z_{k}-\hat{I}_{k}\right)+\left(1-p_{1}\right) \tilde{b}_{k}^{(0)} \\
& =Z_{k}-p_{1} \hat{I}_{k}
\end{aligned}
$$

where $\tilde{b}_{k}^{(1)}$ denotes the soft decision of user $k$ at the GR output with the first stage of PPIC and

$$
\begin{aligned}
\widehat{I}_{k} & =\sum_{j=1, j \neq k}^{K} S_{j} \hat{b}_{j}^{(0)} \rho_{k j} \\
& =\sum_{j=1, j \neq k}^{K} h_{j} A_{j} \hat{b}_{j}^{(0)} \rho_{k j} \\
& =\sum_{j=1, j \neq k}^{K} \alpha_{j}^{2} A_{j} \hat{b}_{j}^{(0)} \rho_{k j}
\end{aligned}
$$

is the estimated MAI using a hard decision.

\section{Performance Analysis}

3.1. Symbol Error Rate Expression. Let $q_{k}$ denote the instantaneous SNR per symbol of the $k$ th quadrature branch $(k=$ $1, \ldots, 2 N)$ at the GR output under quadrature subbranch HS/MRC and HS/MRC schemes. In line with $[2,23]$ and (1)(8) and (10), the instantaneous SNR $q_{k}$ can be defined in the following form:

$$
q_{k}=\frac{E_{b} \alpha_{k}^{2}}{2 \sigma_{n}^{2}}
$$

where $E_{b}$ is the average symbol energy of the transmitted signal $s(t)$.

Assume that we choose $2 L(1 \leq L \leq N)$ quadrature branched out of the $2 \mathrm{~N}$ branches. Then, the SNR per symbol at the GR output under quadrature subbranch HS/MRC and HS/MRC schemes may be presented as

$$
q_{\mathrm{QBHS} / \mathrm{MRC}}=\sum_{k=1}^{2 L} q_{(k)},
$$

where $q_{(k)}$ are the ordered instantaneous SNRs $q_{k}$ and satisfy the following condition

$$
q_{(1)} \geq q_{(2)} \geq \cdots \geq q_{(2 N)}
$$

When $L=N$, we obtain the MRC, as expected. 
Using the moment generating function (MGF) method discussed in $[10,18]$, SER of $M$-ary pulse amplitude modulation (PAM) system conditioned on $q_{\mathrm{QBHS}} \mathrm{MRC}$ is given by

$$
\begin{aligned}
& P_{s}\left(q_{\mathrm{QBHS} / \mathrm{MRC}}\right) \\
& \quad=\frac{2(M-1)}{M \pi} \int_{0}^{0.5 \pi} \exp \left(-\frac{g_{M-\mathrm{PAM}}}{\sin ^{2} \theta} q_{\mathrm{QBHS} / \mathrm{MRC}}\right) d \theta,
\end{aligned}
$$

where

$$
g_{M-\mathrm{PAM}}=\frac{3}{M^{2}-1}
$$

Averaging (38) over $q_{\mathrm{QBH}} \mathrm{MRC}$, the SER of $M$-ary PAM system is determined in the following form:

$$
\begin{aligned}
P_{s} & =\frac{2(M-1)}{M \pi} \int_{0}^{0.5 \pi} \int_{0}^{\infty} \exp \left(-\frac{g_{M-\mathrm{PAM}}}{\sin ^{2} \theta} q\right) f_{q_{\mathrm{QBHS} / \mathrm{MRC}}}(q) d q d \theta \\
& =\frac{2(M-1)}{M \pi} \int_{0}^{0.5 \pi} \varphi_{q_{\mathrm{QBHS} / \mathrm{MRC}}}\left(-\frac{g_{M-\mathrm{PAM}}}{\sin ^{2} \theta}\right) d \theta
\end{aligned}
$$

where

$$
\varphi_{q}(s)=E_{q}\{\exp (s q)\}
$$

is the MGF of random variable $q, E_{q}\{\cdot\}$ is the mathematical expectation of MGF with respect to SNR per symbol. A finite-limit integral for the Gaussian Q-function, which is convenient for numerical integrations is given by [32]

$$
Q(x)=\left\{\begin{array}{l}
\frac{1}{\pi} \int_{0}^{0.5 \pi} \exp \left\{-\frac{x^{2}}{2 \sin ^{2} \theta}\right\} d \theta, \quad x \geq 0, \\
1-\frac{1}{\pi} \int_{0}^{0.5 \pi} \exp \left\{-\frac{x^{2}}{2 \sin ^{2} \theta}\right\} d \theta, \quad x<0 .
\end{array}\right.
$$

The error function can be related to the Gaussian Q-function by

$$
\begin{aligned}
\operatorname{erf}(x) & =\frac{2}{\sqrt{\pi}} \int_{0}^{x} \exp \left(-t^{2}\right) d t \\
& =1-2 Q(\sqrt{2} x)
\end{aligned}
$$

The complementary error function is defined as $\operatorname{erfc}(x)=$ $1-\operatorname{erf}(x)$ so that

$$
Q(x)=\frac{1}{2} \operatorname{erfc}\left(\frac{x}{\sqrt{2}}\right) \quad \text { or } \quad \operatorname{erfc}(x)=2 Q(\sqrt{2} x)
$$

which is convenient for computing values using MATLAB since erfc is a subprogram in MATLAB but the Gaussian Qfunction is not (unless you have a Communications Toolbox). Note that the Gaussian Q-function is the tabulated function.

Now, let us compare (38) and (42) to obtain the closed form expression for the SER of $M$-ary PAM system employing the GR with quadrature subbranch HS/MRC and HS/MRC schemes. We can easily see that taking into account (14), (15), (35), (36), and (39), the SER of $M$-ary PAM system employing the GR with quadrature subbranch HS/MRC and HS/MRC schemes can be defined in the following form

$$
P_{s}\left(q_{\mathrm{QBBHS} / \mathrm{MRC}}\right)=\frac{2 M-1}{M} Q\left(\sqrt{\frac{6}{M^{2}-1} q_{\mathrm{QBHS} / \mathrm{MRC}}}\right) .
$$

Thus, we obtain the closed form expression for the SER of $M$-ary PAM system employing the GR with quadrature subbranch HS/MRC and HS/MRC schemes that agrees with (8.136) and (8.138) in [33]. If $M=2$, the average BER performance of coherent binary phase-shift keying (BPSK) system using the quadrature subbranch HS/MRC and HS/MRC schemes under GR implementation can be determined in the following form:

$$
P_{b}=\frac{1}{\pi} \int_{0}^{0.5 \pi} \varphi_{q_{\mathrm{QBHS} / \mathrm{MRC}}}\left(-\frac{1}{\sin ^{2} \theta}\right) d \theta .
$$

3.2. $M G F$ of $q_{\mathrm{QBH}}$ /MRC. Since all of the $2 N$ quadrature branches are i.i.d., the MGF of $q_{\mathrm{QBHS}}$ MRC takes the following form [12]:

$$
\begin{aligned}
& \varphi_{q_{\mathrm{QBH} S \mathrm{MRC}}}(s) \\
& =2 L\left(\begin{array}{c}
2 N \\
2 L
\end{array}\right) \int_{0}^{\infty} \exp (s q) f(q)[\varphi(s, q)]^{2 L-1}[F(q)]^{2(N-L)} d q,
\end{aligned}
$$

where $f(q)$ and $F(q)$ are, respectively, the probability density function (pdf) and the cumulative distribution function (cdf) of $q$, the SNR per symbol, for each quadrature branch, and

$$
\varphi(s, q)=\int_{q}^{\infty} \exp (s x) f(x) d x
$$

is the marginal moment generating function (MMGF) of SNR per symbol of a single quadrature branch.

Since $g_{k}$ and $g_{k+N}(k=1, \ldots, N)$ follow a zero-mean Gaussian distribution with the variance $\sigma_{h}^{2}$ given by (14), one can show that $q_{k}$ and $q_{k+N}$ follow the Gamma distribution with pdf given by [26]

$$
f(q)= \begin{cases}\frac{1}{\sqrt{q}} \exp \left(-\frac{q}{\bar{q}}\right) \sqrt{\pi \bar{q}}, & q \geq 0, \\ 0, & q \leq 0,\end{cases}
$$

where

$$
\bar{q}=\frac{E_{b} \sigma_{h}^{2}}{\sigma_{n}^{2}}
$$

is the average SNR per symbol for each diversity branch. The MMGF of SNR per symbol of a single quadrature branch can 
be determined in the following form:

$$
\varphi(s, q)=\frac{1}{\sqrt{1-s \bar{q}}} \operatorname{erfc}\left(\sqrt{\frac{1-s \bar{q}}{\bar{q}} q}\right) .
$$

Moreover, the cdf of $q$ becomes

$$
F(q)=1-\varphi(0, q)=1-\operatorname{erfc}\left(\sqrt{\frac{q}{\bar{q}}}\right) .
$$

\section{PCF Determination}

4.1. AWGN Channel. In this section, we determine the PCF at the GR output with the first stage of PPIC. From [20], the linear minimum mean square error (MMSE) solution of PCF for the first stage of PPIC is given by

$$
p_{1, \mathrm{opt}}=\frac{\sigma_{2,0}^{2}-\rho_{1} \sigma_{1,1} \sigma_{2,0}}{\sigma_{1,1}^{2}+\sigma_{2,0}^{2}-2 \rho_{1} \sigma_{1,1} \sigma_{2,0}},
$$

where

$$
\sigma_{1,1}^{2}=E\left\{\left(I_{k}+\zeta_{k}-\hat{I}_{k}\right)^{2}\right\}
$$

is the power of residual MAI plus the total noise component forming at the GR output at the first stage,

$$
\sigma_{2,0}^{2}=E\left\{\left(I_{k}+\zeta_{k}\right)^{2}\right\}
$$

is the power of true MAI plus the total noise component forming at the GR output (also called the 0th stage), and

$$
\rho_{1} \sigma_{1,1} \sigma_{2,0}=E\left\{\left(I_{k}+\zeta_{k}-\widehat{I}_{k}\right)\left(I_{k}+\zeta_{k}\right)\right\}
$$

is a correlation between these two MAI terms. It can be rewritten as

$$
\begin{aligned}
p_{1, \mathrm{opt}}= & \frac{E\left\{\left(I_{k}+\zeta_{k}\right) \hat{I}_{k}\right\}}{E\left\{\hat{I}_{k}^{2}\right\}} \\
= & \frac{1}{(1 / N) \sum_{u \neq l}^{K} S_{u}^{2}+\sum_{u \neq l}^{K} \sum_{v \neq l, u}^{K} S_{u} S_{v} E\left\{\rho_{u l} \rho_{v l} \hat{b}_{u}^{(0)} \hat{b}_{v}^{(0)}\right\}} \\
& \times\left\{\frac{1}{N} \sum_{u \neq l}^{K} A_{u}^{2}\left(1-2 P_{e, u}\right)+\sum_{u \neq l}^{K} \sum_{v \neq l, u}^{K} S_{u} S_{v} E\left\{\rho_{u l} \rho_{v l} \hat{b}_{u}^{(0)} \hat{b}_{v}^{(0)}\right\}+\sum_{v \neq l}^{K} S_{v} E\left\{\rho_{v l} \zeta \hat{b}_{v}^{(0)}\right\}\right\} \\
= & \frac{E\left\{\left[\sum_{j=1, j \neq k}^{K} \alpha_{j}^{2} A_{j} b_{j} \rho_{k j}+\int_{0}^{T_{b}} \alpha_{k}^{2}\left[v_{k_{\mathrm{AF}}}^{2}(t)-v_{k_{\mathrm{PF}}}^{2}(t)\right] d t\right] \sum_{j=1, j \neq k}^{K} \alpha_{j}^{2} A_{j} \hat{b}_{j}^{(0)} \rho_{k j}\right\}}{E\left\{\left[\sum_{j=1, j \neq k}^{K} \alpha_{j}^{2} A_{j} \hat{b}_{j}^{(0)} \rho_{k j}\right]^{2}\right\}} \\
& \times\left\{\frac{1}{N} \sum_{i \neq k}^{K} \alpha_{i}^{4} A_{i}^{2}\left(1-2 P_{e, i}\right)+\sum_{i \neq k}^{K} \sum_{j \neq k, i}^{K} \alpha_{i}^{2} A_{i} \alpha_{j}^{2} A_{j} E\left\{\rho_{i k} \rho_{j k} \hat{b}_{i}^{(0)} \hat{b}_{j}^{(0)}\right\}\right. \\
& \left.+\sum_{j \neq k}^{K} \alpha_{j}^{2} A_{j} E\left[\rho_{j k} \hat{b}_{j}^{(0)} \int_{0}^{T_{b}} \alpha_{k}^{2}\left[v_{k_{\mathrm{AF}}}^{2}(t)-v_{k_{\mathrm{PF}}}^{2}(t)\right] d t\right]\right\},
\end{aligned}
$$

where $P_{e, i}$ is the BER of user $i$ at the corresponding GR output;

$$
E\left\{\hat{b}_{i}^{(0)} \hat{b}_{i}^{(0)}\right\}=1-2 P_{e, i}, \quad E\left\{\rho_{i k}^{2}\right\}=N^{-1} .
$$

The PCF $p_{1, \text { opt }}$ can be regarded as the normalized correlation between the true MAI plus the total noise component forming at the GR output and the estimated MAI. Assume that

$$
\mathbf{b}=\left\{b_{k}\right\}_{k=1}^{K}
$$

is the dataset of all users;

$$
\boldsymbol{\rho}=\left\{\rho_{i k}\right\}_{i, k=1}^{K}
$$

is the correlation coefficient set of random sequences;

$$
f_{\tilde{b}_{i}^{(0)} \mid \mathbf{b}, \boldsymbol{\rho}}\left(\widetilde{b}_{i}^{(0)} \mid \mathbf{b}, \boldsymbol{\rho}\right)=\mathcal{N}\left(E\left\{\widetilde{b}_{i}^{(0)} \mid \mathbf{b}, \boldsymbol{\rho}\right\}, 4 \alpha^{4} \sigma_{n}^{4}\right)
$$

is the conditional normal pdf of $\tilde{b}_{i}^{(0)}$ given $\mathbf{b}$ and $\rho$ and $f_{\tilde{b}_{i}^{(0)}, \tilde{b}_{j}^{(0)} \mid \mathbf{b}, \boldsymbol{\rho}}\left(\tilde{b}_{i}^{(0)}, \tilde{b}_{j}^{(0)} \mid \mathbf{b}, \boldsymbol{\rho}\right)$ is the conditional joint normal pdf of $\tilde{b}_{i}^{(0)}$ and $\tilde{b}_{j}^{(0)}$ given $\mathbf{b}$ and $\boldsymbol{\rho}$. 
Following the derivations in [20], the expectation terms with hard decisions in (57) can be evaluated based on Price's theorem [26] as follows

$$
\begin{gathered}
E\left\{\rho_{i k} \rho_{j k} \hat{b}_{i}^{(0)} \hat{b}_{j}^{(0)}\right\} \\
=E\left\{E\left\{E\left\{\rho_{i k} \rho_{j k} \hat{b}_{i}^{(0)} \hat{b}_{j}^{(0)} \mid \mathbf{b}, \boldsymbol{\rho}\right\} \mid \boldsymbol{\rho}\right\}\right\} \\
=E\left\{E\left\{\rho_{i k} \rho_{j k} \hat{b}_{i}^{(0)}\left(2 Q_{j}-1\right) \mid \boldsymbol{\rho}\right\}\right\}, \\
E\left\{\rho_{j k} \zeta_{k} \hat{b}_{j}^{(0)}\right\} \\
=E\left\{E\left\{E\left\{\rho_{j k} \zeta_{k} \hat{b}_{j}^{(0)} \mid \mathbf{b}, \boldsymbol{\rho}\right\} \mid \boldsymbol{\rho}\right\}\right\} \\
=4 \alpha^{4} \sigma_{n}^{4} E\left\{E\left\{\rho_{j k}^{2} f_{\tilde{b}_{j}^{(0)} \mid \mathbf{b}, \boldsymbol{\rho}}(0 \mid \mathbf{b}, \boldsymbol{\rho}) \mid \boldsymbol{\rho}\right\}\right\}, \\
E\left\{\rho_{u l} \rho_{v l} \hat{b}_{u}^{(0)} \hat{b}_{v}^{(0)}\right\} \\
=E\left\{E\left\{E\left\{\rho_{i k} \rho_{j k} \hat{b}_{i}^{(0)} \hat{b}_{j}^{(0)} \mid \mathbf{b}, \boldsymbol{\rho}\right\} \mid \boldsymbol{\rho}\right\}\right\} \\
=E\left\{E \left\{\rho _ { i k } \rho _ { j k } \left[16 \rho_{i j} \alpha^{4} \sigma_{n}^{4} \tilde{b}_{i}^{(0)}, \tilde{b}_{j}^{(0)} \mid \mathbf{b}, \boldsymbol{\rho}\right.\right.\right. \\
(0,0 \mid \mathbf{b}, \boldsymbol{\rho}) \\
\left.\left.\left.\quad+\left(2 Q_{i}-1\right)\left(2 Q_{j}-1\right)\right] \mid \boldsymbol{\rho}\right\}\right\},
\end{gathered}
$$

where

$$
\begin{gathered}
Q_{k}=Q\left(-\frac{M\left\{\tilde{b}_{k}^{(0)} \mid \mathbf{b}, \boldsymbol{\rho}\right\}}{2 \alpha^{2} \sigma_{n}^{2}}\right), \\
\operatorname{var}\left\{\zeta_{k}\right\}=4 \alpha_{k}^{4} \sigma_{n}^{4}
\end{gathered}
$$

is the total background noise variance forming at the GR output taking into account multipath fading channel; $\sigma_{n}^{2}$ is the additive Gaussian noise variance forming at the PF and AF outputs of GR linear tract; the Gaussian $Q$-function is given by (42).

Although numerical integration in $[20,21]$ can be adopted for determining the optimal PCF $p_{1, \text { opt }}$ for the first stage based on (57)-(64), it requires huge computational complexity. To simplify this problem, we assume that the total background noise forming at the GR output can be considered as a constant factor and may be small enough such that the $Q$ functions in (62) and (64) are all constants and (63) can be approximated to zero. That is

$$
4 \alpha_{k}^{4} \sigma_{n}^{4} \ll \min _{\left\{\alpha_{k} A_{k}, \boldsymbol{\rho}\right\}}\left(E\left\{\tilde{b}_{i}^{(0)} \mid \mathbf{b}, \boldsymbol{\rho}\right\}\right)^{2}=4 \alpha_{m}^{4} A_{m}^{2} N^{-2},
$$

where [34]

$$
\begin{gathered}
\alpha_{m}^{2} A_{m}=\min \alpha_{k}^{2} A_{k} ; \\
\sum_{k \neq m}^{K} \alpha_{k}^{2} A_{k} b_{k} \rho_{k l}=-\alpha_{m}^{2} A_{m} b_{m} \rho_{m k}^{\prime} ; \\
\min \left|\rho_{m k}-\rho_{m k}^{\prime}\right|=\frac{2}{N} .
\end{gathered}
$$

With this, we can rewrite (62) and (64) as follows:

$$
\begin{aligned}
& E\left\{E\left\{\rho_{i k} \rho_{j k} \hat{b}_{i}^{(0)}\left(2 Q_{j}-1\right) \mid \boldsymbol{\rho}\right\}\right\} \\
& =B_{1} E\left\{\rho_{i k} \rho_{j k}\right\} E\left\{\hat{b}_{i}^{(0)} \mid \boldsymbol{\rho}\right\}=0, \\
& E\left\{E \left\{\rho _ { i k } \rho _ { j k } \left[16 \rho_{i k} \alpha^{4} \sigma_{n}^{4} \tilde{b}_{\tilde{b}_{i}^{(0)}, \tilde{b}_{j}^{(0)} \mid \mathbf{b}, \boldsymbol{\rho}}(0,0 \mid \mathbf{b}, \boldsymbol{\rho})\right.\right.\right. \\
& \left.\left.\left.+\left(2 Q_{i}-1\right)\left(2 Q_{j}-1\right)\right] \mid \boldsymbol{\rho}\right\}\right\} \\
& =E\left\{E\left\{16 \alpha^{4} \sigma_{n}^{4} \rho_{i k} \rho_{j k} \rho_{i j} f_{\widetilde{b}_{i}^{(0)}, \tilde{b}_{j}^{(0)} \mid \mathbf{b}, \boldsymbol{\rho}}(0,0 \mid \mathbf{b}, \boldsymbol{\rho}) \mid \boldsymbol{\rho}\right\}\right\} \\
& +B_{2} E\left\{E\left\{\rho_{i k} \rho_{j k} \mid \boldsymbol{\rho}\right\}\right\} \\
& =E\left\{E\left\{4 \alpha^{4} \sigma_{n}^{4} \rho_{i k} \rho_{j k} \rho_{i j} f_{\tilde{b}_{i}^{(0)}, \tilde{b}_{j}^{(0)} \mid \mathbf{b}, \boldsymbol{\rho}}(0,0 \mid \mathbf{b}, \boldsymbol{\rho}) \mid \boldsymbol{\rho}\right\}\right\},
\end{aligned}
$$

where $B_{1}$ and $B_{2}$ are constants. According to assumptions made above, $f_{\tilde{b}_{u}^{(0)}, b_{v}^{(0)} \mid \mathbf{b}, \boldsymbol{\rho}}(0,0 \mid \mathbf{b}, \boldsymbol{\rho})$ can be expressed by

$$
f_{\tilde{b}_{i}^{(0)}, \tilde{b}_{j}^{(0)} \mid \mathbf{b}, \boldsymbol{\rho}}(0,0 \mid \mathbf{b}, \boldsymbol{\rho})=\frac{\exp \left(-0.5 \mathbf{m}_{b}^{T} \mathbf{B}_{b}^{-1} \mathbf{m}_{b}\right)}{8 \pi \alpha^{4} \sigma_{n}^{4} \sqrt{1-\rho_{i j}^{2}}},
$$

where

$$
\begin{gathered}
\mathbf{m}_{b}=\left[E\left\{\widetilde{b}_{i}^{(0)} \mid \mathbf{b}, \boldsymbol{\rho}\right\}, E\left\{\tilde{b}_{j}^{(0)} \mid \mathbf{b}, \boldsymbol{\rho}\right\}\right]^{T} \\
\mathbf{B}_{b}=E\left\{\left(\widetilde{\mathbf{b}}-\mathbf{m}_{b}\right)\left(\widetilde{\mathbf{b}}-\mathbf{m}_{b}\right)^{T}\right\}
\end{gathered}
$$

with

$$
\widetilde{\mathbf{b}}=\left[\widetilde{b}_{i}^{(0)}, \widetilde{b}_{j}^{(0)}\right]^{T}
$$

Since $\mathbf{B}_{b}^{-1}$ is a positive semidefinite matrix, that is,

$$
\mathbf{m}_{b}^{T} \mathbf{B}_{b}^{-1} \mathbf{m}_{b} \geq 0
$$

we can have

$$
0<f_{\tilde{b}_{i}^{(0)}, \tilde{b}_{j}^{(0)} \mid \mathbf{b}, \boldsymbol{\rho}}(0,0 \mid \mathbf{b}, \boldsymbol{\rho}) \leq \max _{\substack{\rho_{i j} \\ \rho_{i j} \neq \pm 1}} \frac{1}{8 \pi \alpha^{4} \sigma_{n}^{4} \sqrt{1-\rho_{i j}^{2}}} .
$$

With the above results,

$$
\min _{\rho_{i j}, \rho_{i j} \neq \pm 1} \sqrt{1-\rho_{i j}^{2}}=\frac{2 \sqrt{N-1}}{N},
$$

where [34]

$$
\begin{aligned}
\rho_{i j} & =1-2 N^{-1} \text { or }-1+2 N^{-1}, \\
E\left\{\rho_{i k} \rho_{j k} \rho_{i j}\right\} & =\sum_{m=1}^{N} \sum_{p=1}^{N} \sum_{q=1}^{N} E\left\{c_{i m} c_{k m} c_{j p} c_{k p} c_{i q} c_{j q}\right\} \\
& =\sum_{m=1}^{N} N^{-3}=N^{-2} .
\end{aligned}
$$


Thus, we can derive a range of $p_{1, \text { opt }}$ as follows:

$$
\begin{gathered}
\frac{\sum_{i \neq k}^{K} \alpha_{i}^{4} A_{i}^{2}\left(1-2 P_{e, u}\right)}{\sum_{i \neq k}^{K} \alpha_{i}^{4} A_{i}^{2}+(1 /(\pi \sqrt{N-1})) \sum_{i \neq k}^{K} \sum_{j \neq l, i}^{K} \alpha_{i}^{2} A_{i} \alpha_{j}^{2} A_{j}} \\
\leq p_{1, \mathrm{opt}}<1-\frac{2 \sum_{i \neq k}^{K} \alpha_{i}^{4} A_{i}^{2} P_{e, u}}{\sum_{i \neq k}^{K} \alpha_{i}^{4} A_{i}^{2}} .
\end{gathered}
$$

If the power control is perfect, that is,

$$
\alpha_{i}^{2} A_{i}=\alpha_{j}^{2} A_{j}=\alpha^{2} A, \quad P_{e, i}=P_{e}
$$

and $P_{e}$ is approximated by the BER of high SNR case, that is, the $Q(\sqrt{N /(K-1)})$ function $[35,36],(77)$ can be rewritten as

$$
\frac{1-2 Q(\sqrt{N /(K-1)})}{1+(K-2) /(\pi \sqrt{N-1})} \leq p_{1, \mathrm{opt}}<1-2 Q\left(\sqrt{\frac{N}{K-1}}\right)
$$

It is interesting to see that the lower and upper boundary values can be explicitly calculated from the processing gain $N$ and the number of users $K$.

4.2. Multipath Channel. Based on representation in (8), we can obtain the received signal vector in the following form:

$$
\mathbf{x}(t)=\sum_{k=1}^{K} A_{k}(t) b_{k} \mathbf{h}_{k}+\mathbf{n}(t)
$$

Introduce the following notation for the correlation coefficient

$$
\omega_{j k}=\mathbf{h}_{j}^{T} \mathbf{h}_{k}, \quad \omega_{k}=\omega_{k k} .
$$

In commercial DS-CDMA systems, the users' spreading codes are often modulated with another code having a very long period. As far as the received signal is concerned, the spreading code is not periodic. In other words, there will be many possible spreading codes for each user. If we use the result derived above, we then have to calculate the optimum PCFs for each possible code and the computational complexity will become very high. Since the period of the modulating code is usually very long, we can treat the code chips as independent random variables and approximate the correlation coefficient $\omega_{j k}$ given by (81) as a Gaussian random variable.

In this case, the GR output for the first stage can be presented in the following form:

$$
\begin{aligned}
Z_{k}(t) & =A_{k}(t) b_{k} \mathbf{h}_{k}^{T} \mathbf{h}_{k}+\sum_{j=1, j \neq k}^{K} A_{j}(t) b_{j} \mathbf{h}_{j}^{T} \mathbf{h}_{k}+\zeta_{k}(t) \\
& =A_{k}(t) b_{k}{\omega_{k}}+\sum_{j=1, j \neq k}^{K} A_{j}(t) b_{j}{\varpi_{k}}+\zeta_{k}(t),
\end{aligned}
$$

where the background noise $\zeta_{k}(t)$ forming at the GR output is given by (30).

Evaluating the GR output process given by (82), based on the well-know results, for example, discussed in [37], we can define the BER performance for the user $k$ in the following form:

$$
\begin{aligned}
P_{b}^{(k)} & =0.5 P\left(Z_{k} \mid b_{k}=1\right)+0.5 P\left(Z_{k} \mid b_{k}=-1\right) \\
& =P\left(Z_{k} \mid b_{k}=1\right) .
\end{aligned}
$$

In (83), we assume that the occurrence probabilities for $b_{k}=$ 1 and $b_{k}=-1$ are equal, and that the error probabilities for $b_{k}=1$ and $b_{k}=-1$ are also equal. As we can see from (82), there are three terms. The first term corresponds to the desired user bit. If we let $b_{k}=1$, it is a deterministic value. The third term in (82) given by (30) corresponds to the GR background noise interference which pdf is defined in [2, Chapter 3, pages 250-263, 324-328]. The second term in (82) corresponds to the interference from other users and is subjected to the binomial distribution. Note that correlation coefficients in (82) are small and DS-CDMA systems are usually operated in low SNR environments. The variance of the second term is then much smaller in comparison with the variance of the third term. Thus, we can assume that $Z_{k}$ conditioned on $b_{k}=1$ can be approximated by Gaussian distribution, as shown in [2, Chapter 3, pages 250-263, 324328] and [31]. Then, the BER performance takes a form

$$
P\left(Z_{k}\right)=Q\left\{\sqrt{\left.\frac{E_{\mathcal{L}}\left\{\mathcal{M}_{k}^{(l)}\right\}}{E_{\mathcal{L}}\left\{\mathcal{V}_{k}^{(l)}\right\}}\right\}},\right.
$$

where $E_{\mathcal{L}}\{\cdot\}$ denotes the expectation operator over the spreading code set $\mathcal{L}$ and $\mathcal{M}_{k}^{(l)}, \mathcal{V}_{k}^{(l)}$ are the expected squared mean and variance of $Z_{k}$, respectively, given the $l$ th possible code in $\mathcal{L}$. Letting

$$
R_{k}=\sum_{j \neq k} q_{j}, \quad \Lambda_{k}=\sum_{j \neq k} \omega_{j k}^{2},
$$

where $q_{j}$ is defined in (35), considering $\omega_{j k}$ as a Gaussian random variable, we obtain

$$
\begin{aligned}
E_{\mathcal{L}}\left\{\mathcal{M}_{k}^{(l)}\right\} & =A_{k}^{2}\left(E_{\mathcal{L}}\left\{\omega_{k}^{(l)}\right\}-p_{k} E_{\mathcal{L}}\left\{\Lambda_{k}^{(l)}\right\}\right)^{2} \\
& =A_{k}^{2}\left(1-p_{k} E_{\mathcal{L}}\left\{\Lambda_{k}^{(l)}\right\}\right)^{2},
\end{aligned}
$$

and the variance as

$E_{\mathcal{L}}\left\{\mathcal{V}_{k}^{(l)}\right\}=4 \sigma_{n}^{4}\left(E_{\mathcal{L}}\left\{\Omega_{1, k}^{(l)}\right\} p_{k}^{2}-2 E_{\mathcal{L}}\left\{\Omega_{2, k}^{(l)}\right\} p_{k}+E_{\mathcal{L}}\left\{\Omega_{3, k}^{(l)}\right\}\right)$.

Note that the expectations in (86) and (87) are operated on interfering user bits and noise using the correlation 
coefficient $\varrho_{j k}$ given by (81). The coefficients of $E_{\mathcal{L}}\left\{\mathcal{V}_{k}^{(l)}\right\}$ are represented by

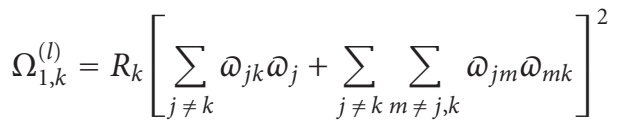

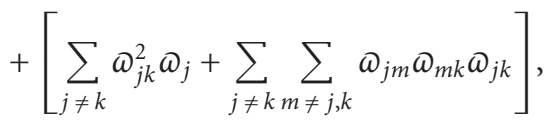

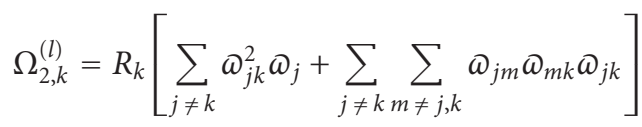

$$
\begin{aligned}
& +\sum_{j \neq k} \omega_{j k}^{2} \text {, } \\
& \Omega_{3, k}^{(l)}=R_{k} \sum_{j \neq k} \omega_{j k}^{2}+\omega_{k} .
\end{aligned}
$$

The optimal PCF for the user $k$ can be found as

$$
\begin{aligned}
p_{k, \mathrm{opt}}= & \arg \max _{p_{k}}\left\{\frac{E_{\mathcal{L}}\left\{\mathcal{M}_{k}^{(l)}\right\}}{E_{\mathcal{L}}\left\{\mathcal{V}_{k}^{(l)}\right\}}\right\} \\
= & \left\{p_{k, \mathrm{opt}}: E_{\mathcal{L}}\left\{\mathcal{V}_{k}^{(l)}\right\} \frac{d E_{\mathcal{L}}\left\{\mathcal{M}_{k}^{(l)}\right\}}{d p_{k}}\right. \\
& \left.-E_{\mathcal{L}}\left\{\mathcal{M}_{k}^{(l)}\right\} \frac{d E_{\mathcal{L}}\left\{\mathcal{V}_{k}^{(l)}\right\}}{d p_{k}}=0\right\} .
\end{aligned}
$$

Substituting (86)-(90) into (91) and simplifying the result, we obtain the following equation

$$
p_{k, \text { opt }}=\frac{E_{\mathscr{L}}\left\{\Omega_{2, k}^{(l)}\right\}-E_{\mathscr{L}}\left\{\Omega_{3, k}^{(l)}\right\} E_{\mathcal{L}}\left\{\Lambda_{k}^{(l)}\right\}}{E_{\mathscr{L}}\left\{\Omega_{1, k}^{(l)}\right\}-E_{\mathscr{L}}\left\{\Omega_{2, k}^{(l)}\right\} E_{\mathscr{L}}\left\{\Lambda_{k}^{(l)}\right\}} .
$$

Unlike that in AWGN channel, the result for the aperiodic code scenario is more difficult to obtain because there are more correlation terms in (85)-(91) to work with. Before evaluation of the expectation terms in (92), we define some function as follows:

$$
\begin{gathered}
\alpha_{j k}(m, n)=\alpha_{j, m} \alpha_{k, n}, \\
\tau_{j k}(m, n)=\tau_{j, m}-\tau_{k, n}, \\
\psi_{j k}(m, n)=\widetilde{\mathbf{a}}_{j, m}^{T} \tilde{\mathbf{a}}_{k, n} .
\end{gathered}
$$

Thus, (93) define some relative figures between the $m$ th channel path of the $j$ th user and the $n$th channel path of the $k$ th user. The notation $\alpha_{j k}(m, n)$ denotes the path gain product, $\tau_{j k}(m, n)$ is the relative path delay, and $\psi_{j k}(m, n)$ is the code correlation with the relative delay $\tau_{j k}(m, n)$. Expanding (93), we have seven expectation terms to evaluate.
For purpose of illustration, we show how to evaluate the first term, $E_{\mathcal{L}}\left\{\varpi_{j k}^{2}\right\}$ here. By definition, we have $\Theta_{j k}$ as

$$
\begin{aligned}
\varpi_{j k} & =\mathbf{h}_{j}^{T} \mathbf{h}_{k} \\
& =\left\{\sum_{m=1}^{L} \widetilde{\mathbf{a}}_{j, m} \alpha_{j, m}\right\}^{T}\left\{\sum_{n=1}^{L} \widetilde{\mathbf{a}}_{k, n} \alpha_{k, n}\right\} \\
& =\sum_{m=1}^{L} \sum_{n=1}^{L} \alpha_{j, m} \alpha_{k, n} \widetilde{\mathbf{a}}_{j, m}^{T} \widetilde{\mathbf{a}}_{k, n} \\
& =\sum_{m=1}^{L} \sum_{n=1}^{L} \alpha_{j k}(m, n) \psi_{j k}(m, n) .
\end{aligned}
$$

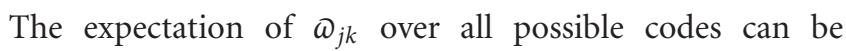
presented in the following form:

$$
\begin{aligned}
& E_{L}\left\{\Phi_{j k}^{2}\right\}=E\left\{\sum_{m_{1}=1}^{L} \sum_{n_{1}=1}^{L} \sum_{m_{2}=1}^{L} \sum_{n_{2}=1}^{L} \alpha_{j k}\left(m_{1}, n_{1}\right)\right. \\
&\left.\times \psi_{j k}\left(m_{1}, n_{1}\right) \alpha_{j k}\left(m_{2}, n_{2}\right) \psi_{j k}\left(m_{2}, n_{2}\right)\right\} \\
&=\sum_{m_{1}=1}^{L} \sum_{n_{1}=1}^{L} \sum_{m_{2}=1}^{L} \sum_{n_{2}=1}^{L} \alpha_{j k}\left(m_{1}, n_{1}\right) \alpha_{j k}\left(m_{2}, n_{2}\right) \\
& \times E\left\{\psi_{j k}\left(m_{1}, n_{1}\right) \psi_{j k}\left(m_{2}, n_{2}\right)\right\} .
\end{aligned}
$$

Introduce the following function

$$
G_{j k}\left(m_{1}, n_{1}, m_{2}, n_{2}\right)=B^{2} E\left\{\psi_{j k}\left(m_{1}, n_{1}\right) \psi_{j k}\left(m_{2}, n_{2}\right)\right\} .
$$

The coefficient $B^{2}$ in (96) is only the normalization constant. Since the spreading codes are seen as random, only if $\tau_{j k}\left(m_{1}, n_{1}\right)$ is equal to $\tau_{j k}\left(m_{2}, n_{2}\right)$ will $G_{j k}\left(m_{1}, n_{1}, m_{2}, n_{2}\right)$ be nonzero. Consider a specific set of $\left\{m_{1}, n_{1}, m_{2}, n_{2}\right\}$ such that

$$
\tau_{j k}\left(m_{1}, n_{1}\right)=\tau_{j k}\left(m_{2}, n_{2}\right)=\tau, \quad \tau \geq 0 .
$$

In this case, we have

$$
G_{j k}\left(m_{1}, n_{1}, m_{2}, n_{2}\right)=B^{2} \sum_{\nu=0}^{N-\tau-1} E\left\{a_{j, v+\tau}^{2} a_{k, v}^{2}\right\}=N-\tau .
$$

At $\tau<0$, we have the same result except that the sign of $\tau$ in (98) is plus. We can conclude that the function $G_{j k}\left(m_{1}, n_{1}, m_{2}, n_{2}\right)$ in (96) can be written in the following form:

$$
\begin{aligned}
& G_{j k}\left(m_{1}, n_{1}, m_{2}, n_{2}\right) \\
& \quad= \begin{cases}N-|\tau|, & \text { if } \tau_{j k}\left(m_{1}, n_{1}\right)=\tau_{j k}\left(m_{2}, n_{2}\right)=\tau \\
0, & \text { otherwise. }\end{cases}
\end{aligned}
$$


Using (95), (96), and (98), we can evaluate $E_{\mathscr{L}}\left\{\varrho_{j k}^{2}\right\}$ in (88)(90). The formulations from the other six expectation terms can be obtain by mathematical transformation that is not difficult.

We now provide a simple example to show the multipath effect on the optimal PCFs. Introduce the following notations that are satisfied for all $k$ :

$$
\boldsymbol{\tau}_{k}=[0, T]^{T}, \quad \boldsymbol{\alpha}_{k}=[\beta, \delta]^{T}, \quad \beta^{2}+\delta^{2}=1 .
$$

Using (100) and taking into consideration that in the case of AWGN channel

$$
E_{\mathscr{L}}\left\{\Lambda_{k}^{(l)}\right\}=\frac{K-1}{N}
$$

at given the $l$ th possible code in $\mathcal{L}$, we can write for the case of the multipath channel

$$
\begin{aligned}
& E_{\mathcal{L}}\left\{\Lambda_{k}^{(l)}\right\}=\frac{K-1}{N}+\frac{2(N-T) \beta^{2} \delta^{2}(K-1)}{N^{2}} \\
& E_{\mathcal{L}}\left\{\Omega_{1, k}^{(l)}\right\} \\
& =E_{\mathcal{L}}\left\{R_{k}^{(l)}\left\{\sum_{j=1, j \neq k}^{K} \rho_{j k}^{(l)}+\sum_{j=1, j \neq k}^{K} \sum_{m=1, m \neq j, k}^{K} \rho_{j m}^{(l)} \rho_{m k}^{(l)}\right\}^{2}\right. \\
& \left.\quad+\sum_{j=1, j \neq k}^{K}\left(\rho_{j k}^{(l)}\right)^{2}+\sum_{j=1, j \neq k}^{K} \sum_{m=1, m \neq j, k}^{K} \rho_{j m}^{(l)} \rho_{m k}^{(l)} \rho_{j k}^{(l)}\right\} \\
& +2(N-T) \beta^{2} \delta^{2} \\
& \quad \times\left\{R _ { k } N ^ { - 4 } \left[N^{2}+10 N+4(N-T) \beta^{2} \delta^{2}\right.\right. \\
& \quad+2(K-2)\left(4 N+3 K+(N-T) \beta^{2} \delta^{2}+1\right] \\
& \left.\quad+(K-1) N^{-3}(N+3 K-2)\right\} \\
& +4(N-2 T) \beta^{4} \delta^{4}\left[R_{k} K N^{-4}+6 K-12\right] \\
& +R_{k} N^{-4}(6 N-10 T) \beta^{4} \delta^{4},
\end{aligned}
$$

$$
\begin{aligned}
& E_{\mathcal{L}}\left\{\Omega_{2, k}^{(l)}\right\} \\
& =E_{\mathcal{L}}\left\{R_{k}^{(l)}\left\{\sum_{j=1, j \neq k}^{K}\left(\rho_{j k}^{(l)}\right)^{2}+\sum_{j=1, j \neq k}^{K} \sum_{m=1, m \neq j, k}^{K} \rho_{j m}^{(l)} \rho_{m k}^{(l)} \rho_{j k}^{(l)}\right\}\right. \\
& \left.\quad+\sum_{j=1, j \neq k}^{K}\left(\rho_{j k}^{(l)}\right)^{2}\right\} \\
& \quad+2(N-T) \beta^{2} \delta^{2}\left[R_{k} N^{-3}(N+3 K-2)+(k-1) N^{-2}\right],
\end{aligned}
$$

$$
\begin{aligned}
E_{\mathcal{L}} & \left\{\Omega_{3, k}^{(l)}\right\} \\
& =E_{\mathcal{L}}\left\{R_{k} \sum_{j=1, j \neq k}^{K}\left(\rho_{j k}^{(l)}\right)^{2}+1\right\}+2(N-T) \beta^{2} \delta^{2} R_{k} N^{-2} .
\end{aligned}
$$

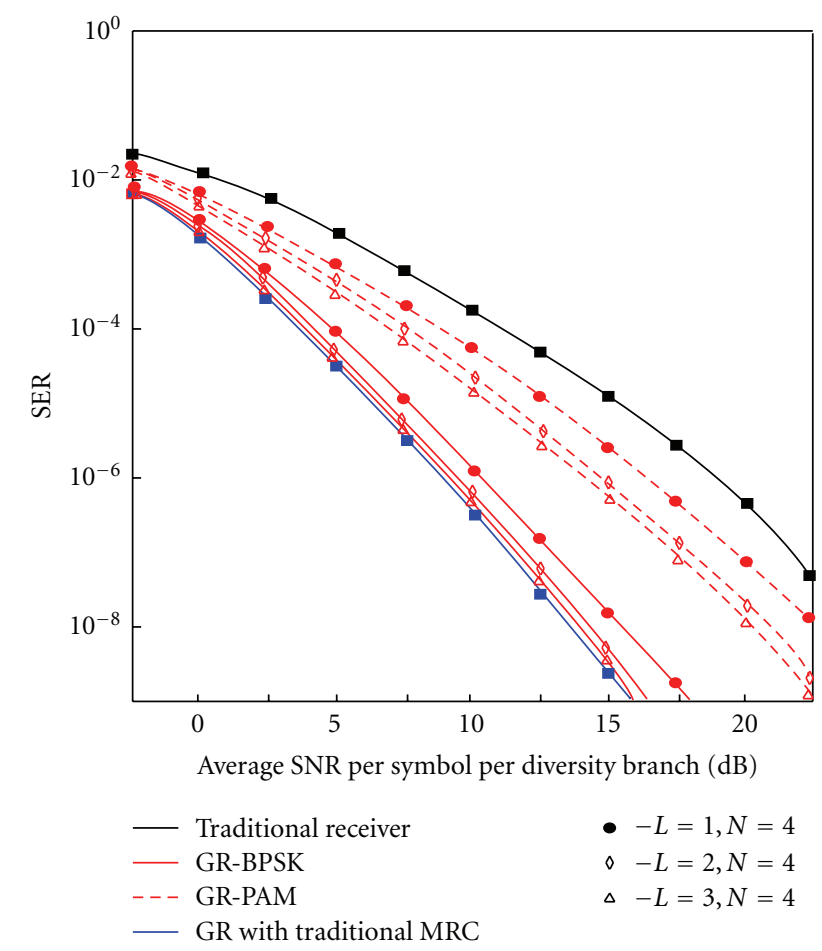

FIgURE 3: Average SER of coherent BPSK and 8-PAM for GR with quadrature subbranch HS/MRC and HS/MRC schemes versus the average SNR per symbol per diversity for various values of $2 L$ with $2 N=8$.

Note that the first terms in (102)-(105) correspond to the optimal PCFs in AWGN channel. Other terms are due to the multipath effect. It is evident to see that if $\delta=0$, we have the case of an AWGN channel.

\section{Simulation Results}

5.1. Selection/Maximal-Ratio Combining. In this section, we discuss some examples of GR performance with quadrature subbranch HS/MRC and HS/MRC schemes and compare with the conventional HS/MRC receiver. The average SER of coherent BPSK and 8-PAM signals under processing by the GR with quadrature subbranch HS/MRC and HS/MRC schemes as a function of average SNR per symbol per diversity branch for various values of $2 L$ and $2 N=8$ is presented in Figure 3. It is seen that the GR SER performance with quadrature subbranch HS/MRC and HS/MRC schemes at $(L, N)=(3,4)$ achieves virtually the same performance as the GR with traditional MRC, and that the performance at $(L, N)=(2,4)$ is typically less than $0.5 \mathrm{~dB}$ in SNR poorer than that of GR with traditional MRC in [19]. Additionally, a comparison with the traditional HS/MTC receiver in $[6,7]$ is made. The advantage of GR implementation in DS-CDMA systems is evident.

The average SER of coherent BPSK and 8-PAM signals under processing by GR with quadrature subbranch HS/MRC and HS/MRC schemes as a function of average SNR per symbol per diversity branch for various values of $2 \mathrm{~N}$ at 


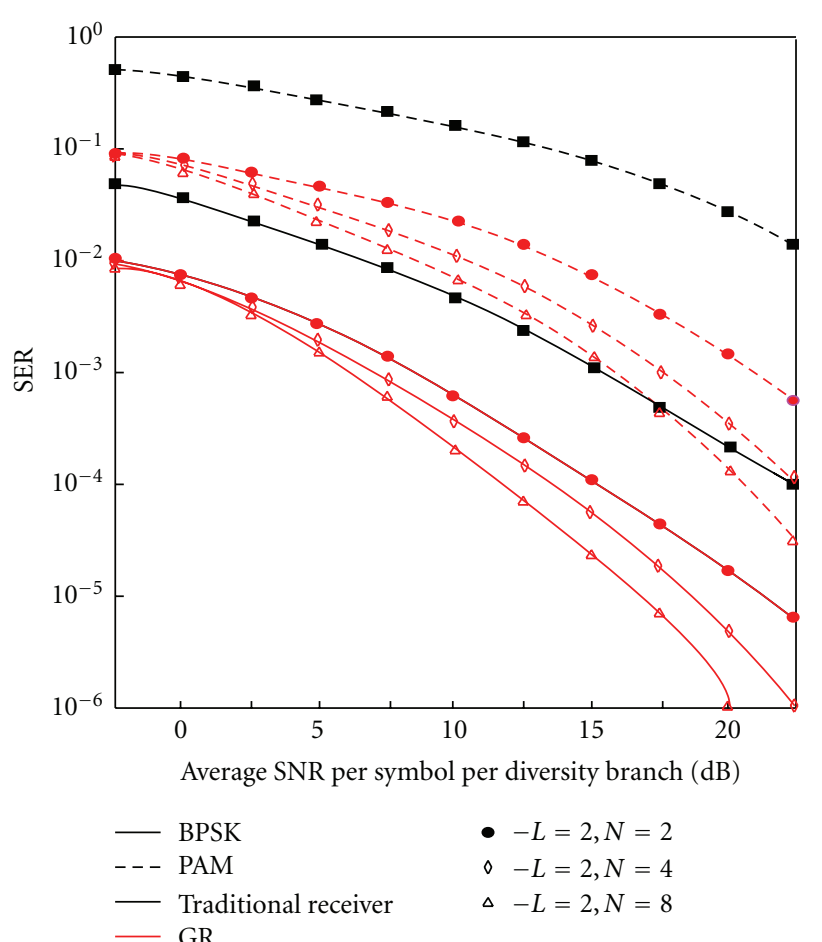

FIGURE 4: Average SER of coherent BPSK and 8-PAM for GR with quadrature subbranch HS/MRC and HS/MRC schemes versus the average SNR per symbol per diversity for various values of $2 \mathrm{~N}$ with $2 L=4$.

$2 L=4$ is shown in Figure 4 . We note the substantial benefits of increasing the number of diversity branches $N$ for fixed $L$. Comparison with the traditional HS/MRC receiver is made. The advantage of GR using is evident.

Comparative analysis of average BER as a function of the average SNR per bit per diversity branch of coherent BPSK signals employing GR with quadrature subbranch HS/MRC and HS/MRC schemes and GR with traditional HS/MRC scheme for various values of $L$ with $N=8$ is presented in Figure 5. To achieve the same value of average SNR per bit per diversity branch, we should choose $2 L$ quadrature branches for the GR with quadrature subbranch HS/MRC, HS/MRC schemes, and $L$ diversity branches for the GR with traditional HS/MRC scheme. Figure 5 shows that the GR BER performance with quadrature subbranch HS/MRC and HS/MRC schemes is much better than that of the GR with traditional HS/MRC scheme, about $0.5 \mathrm{~dB}$ to $1.2 \mathrm{~dB}$, when $L$ is less than one half $N$. This difference decreases with increasing $L$.

This is expected because when $L=N$, we obtain the same performance. Some discussion of increases in GR complexity and power consumption is in order. We first note that GR with quadrature subbranch HS/MRC and $\mathrm{HS} / \mathrm{MRC}$ schemes requires the same number of antennas as GR with traditional HS/MRC scheme. On the other hand, the former requires twice as many comparators as the latter, to select the best signals for further processing. However, GR designs that process the quadrature signal components will

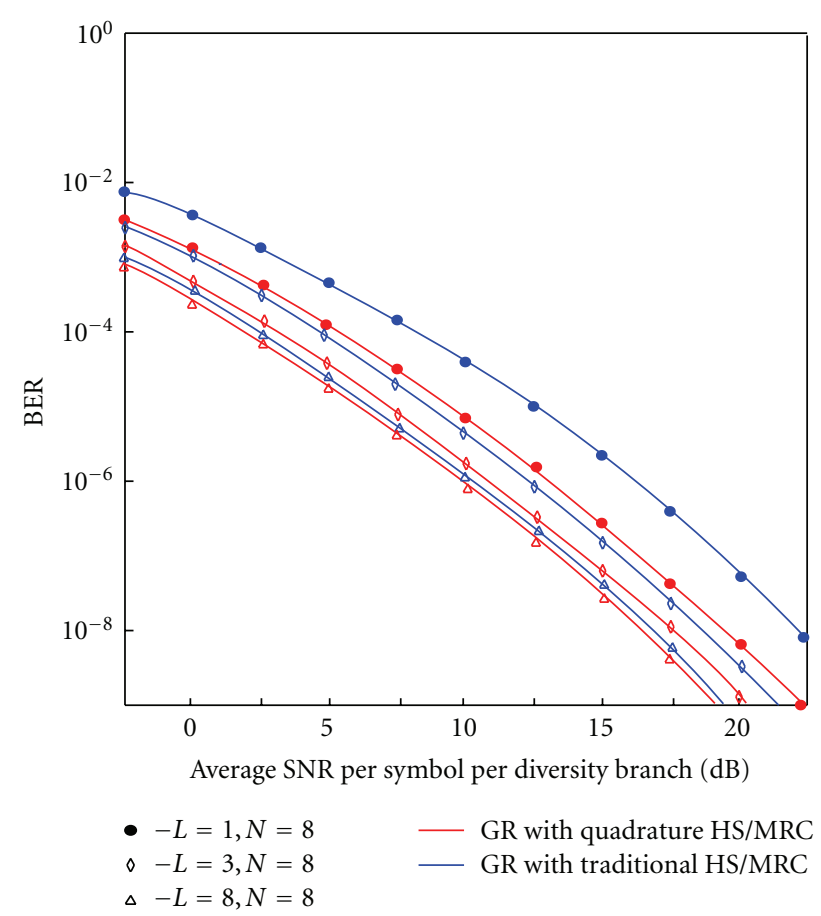

FIGURE 5: Comparison of the average BER of coherent BPSK and 8-PAM for GR with quadrature subbranch HS/MRC and HS/MRC schemes versus the average SNR per symbol per diversity for various values of $2 L$ with $N=8$.

require $2 L$ receiver chains for either the GR with quadrature subbranch HS/MRC and HS/MRC schemes or the GR with traditional HS/MRC scheme. Such receiver designs will use only a little additional power, as GR chains consume much more power than the comparators.

On the other hand, GR designs that implement cophasing of branch signals without splitting the branch signals into the quadrature components will require $L$ receiver chains for GR with traditional HS/MRC scheme and $2 L$ receiver chains for GR with quadrature subbranch HS/MRC and HS/MRC schemes, with corresponding hardware and power consumption increases.

5.2. Synchronous DS-CDMA System. To demonstrate usefulness of the optimal PCF range given by (79), we performed a number of simulations for an asynchronous DS-CDMA system with perfect power control. In simulations, the random spreading codes with length $N=64$ were used for each user, and the number of users was $K=40$ [38]. Figure 6 shows the BER performance of single-stage harddecision GR based on PPIC for different magnitudes of SNR and various values of PCF, where the optimal PCF for the first stage lies between 0.3169 (lower boundary) and 0.7998 (upper boundary). It can be seen that, for all the SNR cases, the GR based on PPIC using the average of the lower and upper boundary values, that is, 0.5584 , as the PCF, has close BER performance to that using the optimal PCF. Additionally, comparison of GR implementation in 


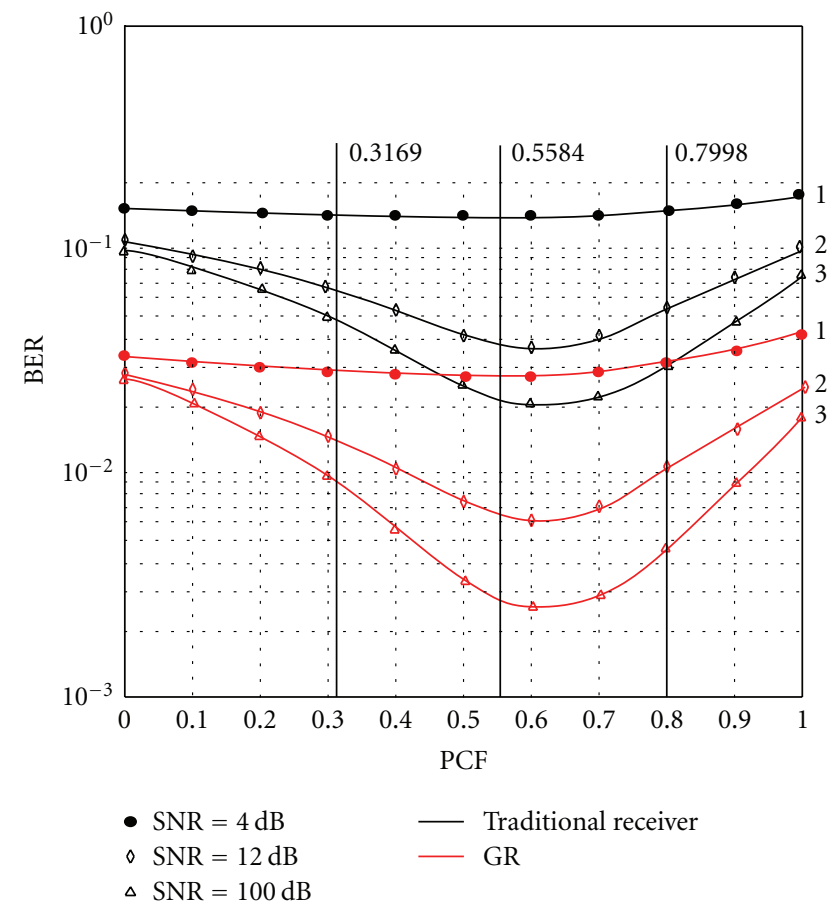

FIGURE 6: The BER performance of the single-state GR based on PPIC with hard decisions for different SNRs and PCFs.

DS-CDMA systems with the conventional detector in [20] is presented. These results show us a great superiority of the GR employment over the conventional detector in [20].

Figure 7 shows the BER performance at each stage for the three-stage GR based on the PPIC using different PCFs at the first stage, that is, the average value and an arbitrary value. PCFs for these two three-stage cases are

$$
\left(a_{1}, a_{2}, a_{3}\right)=(0.5584,0.8,0.9) \text { and }(0.7,0.8,0.9) \text {, }
$$

respectively. The results demonstrate that the BER performances of GR employed by DS-CDMA systems of the cases using the proposed PCF at the first stage outperform ones of GR implemented in DS-CDMA system using arbitrary PCF at the first stage. Furthermore, the GR BER performance at the second stage for the case using the proposed PCF at the first stage achieves the BER performance of the GR comparable to that of the three-stage GR based on PPIC using an arbitrary PCF at the first stage. Comparison between the AWCN and multipath channels is also presented in Figure 7. We see that in the case of multipath channel, the BER performance is deteriorated. This fact can be explained by the additional correlation terms in (102)-(105).

Figure 8 demonstrates the optimal PCF versus the number of users both for the synchronous AWGN and for the multipath channels. We carry out simulation for the AWGN channel under the following conditions: the Gold codes, $\mathrm{SNR}=12 \mathrm{~dB}$, the spreading codes are the periodic, and

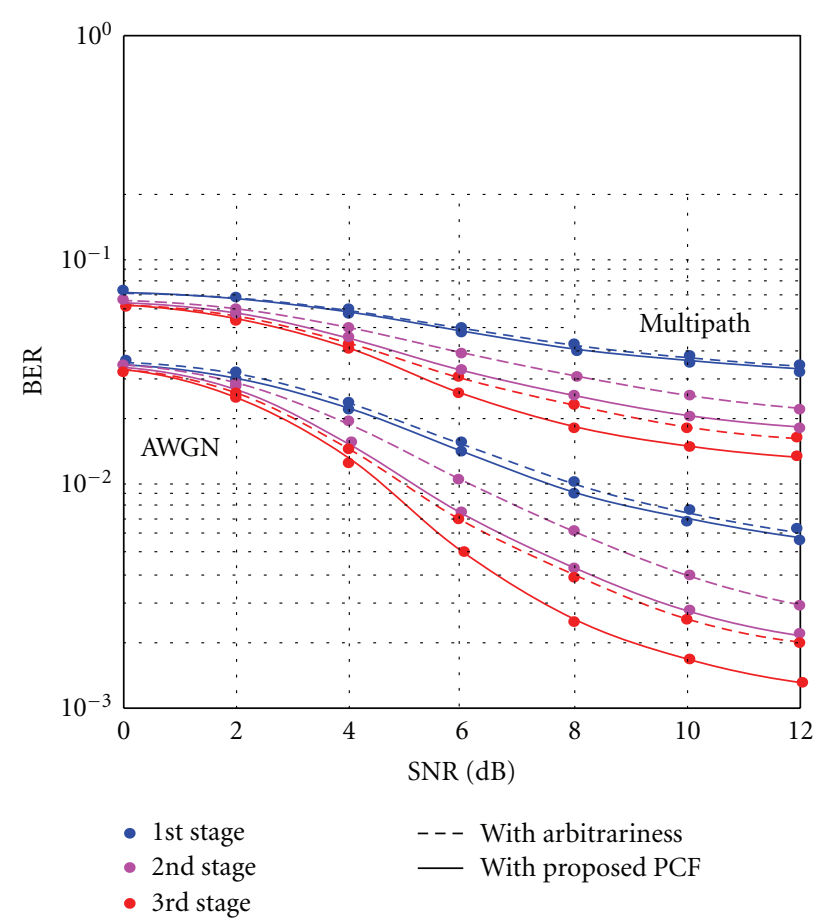

FIGURE 7: The BER performance at each stage for three-stage GR based on the PPIC with hard decisions for different PCFs at the first stage, that is, the average value and an arbitrary value: AWGN and multipath channels.

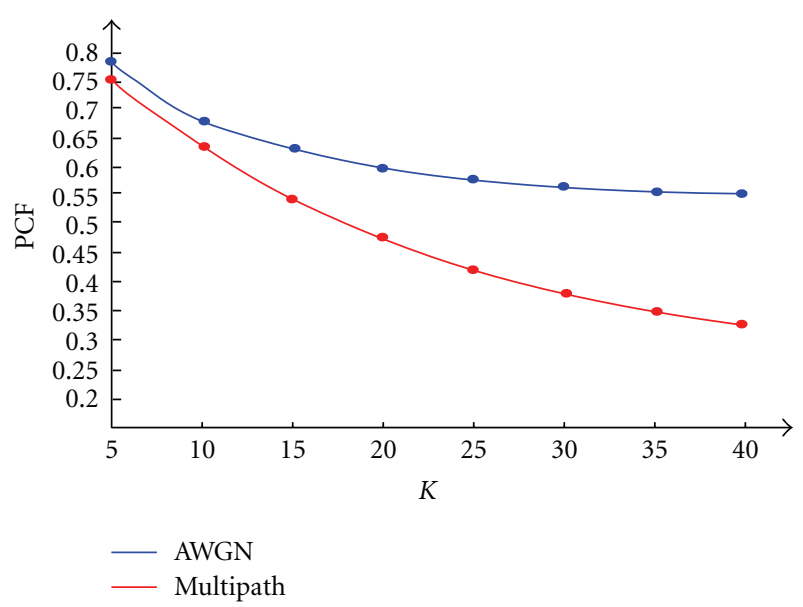

FIGURE 8: Optimal PCF versus the number of users: the AWGN and multipath channels.

perfect power control. The multipath channel assumed is a two-ray channel with the transfer function

$$
W_{k}(Z)=0.762+0.648 Z^{-2}
$$

for all users. In the case of multipath channel, we employ aperiodic codes, $\mathrm{SNR}=12 \mathrm{~dB}$, and perfect power control. 


\section{Conclusions}

The GR performance with quadrature subbranch HS/MRC and HS/MRC schemes for a 1-D signal modulation in Rayleigh fading was investigated. The SER of $M$-ary PAM, including coherent BPSK modulation, was derived. Results show that the GR with quadrature subbranch HS/MRC and HS/MRC schemes performs substantially better the GR with traditional HS/MRC scheme, particularly, when $L$ is smaller than one half $N$, and much better than the traditional $\mathrm{HS} / \mathrm{MRC}$ receiver.

We have also derived the optimal PCF range for GR first stage based on the PPIC, which is employed by DS-CDMA system, with hard decisions in multipath fading channel. Computer simulation shows that the BER performance of the GR employed by DS-CDMA system with multipath fading channel in the case of periodic code scenario and using the average of the lower and upper boundary values is close to that of the GR of the case using the real optimal PCF, whether the SNR is high or low. It has also been shown that GR employment in a DS-CDMA system with multipath fading channel in the case of periodic code scenario allows us to observe a great superiority over the conventional receiver discussed in [20]. The procedure discussed in [20] is also acceptable for GR employment by DS-CDMA systems. It has also been demonstrated that the two-stage GR based on PPIC using the proposed PCF at the first stage achieves such BER performance comparable to that of the three-stage GR based on PPIC using an arbitrary PCF at the first stage. This means that at the same BER performance, the number of stages (or complexity) required for the multistage GR based on PPIC could be reduced when the proposed PCF is used at the first stage. It can be shown that the proposed PCF selection approach is applicable to multipath fading cases at GR employment in DS-CDMA systems even if no perfect power control is assumed, but this is a subject of future work. We have also compared the BER performance at the optimal PCF in the case of AWGN and multipath channels and presented a sensitivity of the BER performance to the values of PCF for both cases.

\section{Acknowledgments}

This paper was supported by Kyungpook National University Research Grant, 2009. Additionally, the author would like to thank the anonymous reviewers for the comments and suggestions that helped to improve the quality of this paper.

\section{References}

[1] V. Tuzlukov, Signal Processing in Noise: A New Methodology, IEC, Minsk, Belarus, 1998.

[2] V. Tuzlukov, Signal Detection Theory, Springer, New York, NY, USA, 2001.

[3] V. Tuzlukov, Signal Processing Noise, CRC Press, Boca Raton, Fla, USA, 2002.

[4] V. Tuzlukov, Signal and Image Processing in Navigational Systems, CRC Press, Boca Raton, Fla, USA, 2004.

[5] V. P. Tuzlukov, "A new approach to signal detection theory," Digital Signal Processing, vol. 8, no. 3, pp. 166-184, 1998.
[6] M. Z. Win and J. H. Winters, "Analysis of hybrid selection/ maximal-ratio combining in Rayleigh fading," IEEE Transactions on Communications, vol. 47, no. 12, pp. 1773-1776, 1999.

[7] M. Z. Win and J. H. Winters, "Virtual branch analysis of symbol error probability for hybrid selection/maximalratio combining in Rayleigh fading," IEEE Transactions on Communications, vol. 49, no. 11, pp. 1926-1934, 2001.

[8] N. Kong and L. B. Milstein, "Average SNR of a generalized diversity selection combining scheme," IEEE Communications Letters, vol. 3, no. 3, pp. 57-79, 1999.

[9] M. S. Alouini and M. K. Simon, "Performance of coherent receivers with hybrid SC/MRC over Nakagami-m fading channels," IEEE Transactions on Vehicular Technology, vol. 48, no. 4, pp. 1155-1164, 1999.

[10] M. S. Alouini and M. K. Simon, "An MGF-based performance analysis of generalized selection combining over Rayleigh fading channels," IEEE Transactions on Communications, vol. 48, no. 3, pp. 401-415, 2000.

[11] R. K. Mallik and M. Z. Win, "Analysis of hybrid selection/maximal-ratio combining in correlated Nakagami fading," IEEE Transactions on Communications, vol. 50, no. 8, pp. 1372-1383, 2002.

[12] A. Annamalai and C. Tellambura, "A new approach to performance evaluation of generalized selection diversity receivers in wireless channels," in Proceedings of the 54th IEEE Vehicular Technology Conference (VTC '01), vol. 4, pp. 23092313, October 2001.

[13] J. G. Proakis, Digital Communications, McGraw-Hill, New York, NY, USA, 4th edition, 2001.

[14] U. Mengali and A. N. D. Andrea, Synchronization Techniques for Digital Receivers, Plenum, New York, NY, USA, 1997.

[15] L. Tong and S. Perreau, "Multichannel blind identification: from subspace to maximum likelihood methods," Proceedings of the IEEE, vol. 86, no. 10, pp. 1951-1968, 1998.

[16] J. K. Tugnait, L. Tong, and Z. Ding, "Single-user channel estimation and equalization," IEEE Signal Processing Magazine, vol. 17, no. 3, pp. 17-28, 2000.

[17] K. Abed-Meraim, W. Qaiu, and Y. Hua, "Blind system identification," Proceedings of the IEEE, vol. 85, no. 8, pp. 13101322, 1997.

[18] M. S. Alouini, "A unified approach for calculating error rates of linearly modulated signals over generalized fading channels," IEEE Transactions on Communications, vol. 47, no. 9, pp. 1324-1334, 1999.

[19] V. Tuzlukov, W.-S. Yoon, and Y. D. Kim, "Wireless sensor networks based on the generalized approach to signal processing with fading channels and receive antenna array," WSEAS Transactions on Circuits and Systems, vol. 10, no. 3, pp. 21492155, 2004.

[20] D. Divsalar, M. K. Simon, and D. Raphaeli, "Improved parallel interference cancellation for CDMA," IEEE Transactions on Communications, vol. 46, no. 2, pp. 258-268, 1998.

[21] P. G. Renucci and B. D. Woerner, "Optimisation of soft interference cancellation for DS-CDMA," Electronics Letters, vol. 34, no. 8, pp. 731-733, 1998.

[22] D. Guo, Linear Parallel Interference Cancellation in CDMA, M.S. thesis, National University of Singapore, 1998.

[23] Y. T. Hsieh and W. R. Wu, "Optimal two-stage decoupled partial PIC receivers for multiuser detection," IEEE Transactions on Wireless Communications, vol. 4, no. 1, pp. 112-127, 2005.

[24] M. Ghotbi and M. R. Soleymani, "Multiuser detection of DSCDMA signals using partial parallel interference cancellation in satellite communications," IEEE Journal on Selected Areas in Communications, vol. 22, no. 3, pp. 584-593, 2004. 
[25] K. M. Wu and C. L. Wang, "Soft-input soft-output partial parallel interference cancellation for DS-CDMA systems," in Proceedings of the International Conference on Communications (ICC '01), pp. 1172-1176, Helsinki, Finland, June 2000.

[26] A. Papoulis and S. U. Pillai, Probability, Random Variables and Stochastic Processes, McGraw-Hill, New York, NY, USA, 4th edition, 2002.

[27] G. Xue, J. F. Weng, T. Le-Ngoc, and S. Tahar, "An analytical model for performance evaluation of parallel interference cancellers in CDMA systems," IEEE Communications Letters, vol. 4, no. 6, pp. 184-186, 2000.

[28] J. H. Kim, V. Tuzlukov, W. S. Yoon, and Y. D. Kim, "Generalized detector under nonorthogonal multipulse modulation in remote sensing systems," WSEAS Transactions on Signal Processing, vol. 2, no. 1, pp. 203-208, 2005.

[29] V. Tuzlukov, W. S. Yoon, and Y. D. Kim, "Adaptive beamformer generalized detector in wireless sensor networks," in Proceedings of the IASTED International Conference on Parallel and Distributed Computing and Networks (PDCN '04), pp. 195-200, Innsbruck, Austria, February 2004.

[30] J. H. Kim, V. Tuzlukov, W. S. Yoon, and Y. D. Kim, "Macrodiversity in wireless sensor networks based on the generalized approach to signal processing," WSEAS Transactions and Communications, vol. 4, no. 8, pp. 648-653, 2005.

[31] V. Tuzlukov, "Multiuser generalized detector for uniformly quantized synchronous CDMA signals in AWGN channels," Telecommunications Review, vol. 20, no. 5, pp. 836-848, 2010.

[32] J. W. Craig, "A new, simple and exact result for calculating the probability of error for two-dimensional signal constellations," in Proceedings of the IEEE Military Communications Conference (MILCOM '91), vol. 2, pp. 571-575, McLean, Va, USA, November 1991.

[33] R. E. Ziemer and W. H. Tranter, Principles of Communications: Systems, Modulation, and Noise, John Wiley \& Sons, New York, NY, USA, 6th edition, 2010.

[34] R. K. Morrow Jr. and J. S. Lehnert, "Bit-to-bit error dependence in slotted DS/SSMA packer systems with random signature sequences," IEEE Transactions on Communications, vol. 37, no. 10, pp. 1052-1061, 1989.

[35] J. C. Liberti Jr. and T. S. Rappaport, Smart Antennas for Wireless Communications: IS-95 and Third Generation CDMA Applications, Prentice Hall, Upper Saddle River, NJ, USA, 1999.

[36] D. R. Brown III and C. R. Johnson Jr., "SINR, power efficiency, and theoretical system capacity of parallel interference cancellation," Journal of Communications and Networks, vol. 3, no. 3, pp. 228-237, 2001.

[37] S. Manohar, V. Tikiya, R. Annavajjala, and A. Chockalingam, "BER-optimal linear parallel interference cancellation for multicarrier DS-CDMA in Rayleigh fading," IEEE Transactions on Communications, vol. 55, no. 6, pp. 1253-1265, 2007.

[38] V. Tuzlukov, "Selection of partial cancellation factors in DS-CDMA systems employing the generalized detector," in Proceedings of the 12th WSEAS International Conference on Communications: New Aspects of Communications, pp. 129134, Heraklion Crete, Greece, July 2008. 